\title{
Erratum: Crystal symmetry based selection rules for anharmonic phonon-phonon scattering from a group theory formalism [Phys. Rev. B 103, 184302 (2021)]
}

\author{
Runqing Yang, Shengying Yue, Yujie Quan, and Bolin Liao $\odot$
}

(Received 30 July 2021; published 3 September 2021)

DOI: 10.1103/PhysRevB.104.099902

We would like to clarify that the space-group selection rules we reviewed in Sec. II of our paper are well-established results in group theory and can be found in extensive previous literature and group-theory textbooks (e.g., Refs. [29]-[32] cited in our paper). The purpose of including this section in our paper was to add more context for readers who are not familiar with the group-theory formalism and we did not intend to claim novelty on this part. Despite our efforts to include proper and extensive references to relevant previous literature, we regret to have left the impression to some readers that we were claiming to have invented a theory that has long existed. The main contribution of our work is to apply the space-group selection rules to anharmonic phonon-phonon scattering with an explicit analysis of graphene as an example, which was further illustrated by a first-principles simulation.

In addition, we would like to correct the last statement of the Introduction section of our paper regarding the work by Dr. A. Cammarata [RSC Adv. 9, 37491 (2019), cited as Ref. [33] in our paper]. In his paper, Dr. Cammarata applied the space-group selection rules to study anharmonic phonon-phonon scattering in a transition metal dichalcogenide. Although not explicitly discussed in his paper, the space-group selection rules are general and can be applied to phonons with specific wave vectors. Thus, our original assessment "the scattering selection rules for phonons with particular wave vectors were not established" was inaccurate.

We thank Dr. Cammarata for raising this issue. 